J. Lake Sci. (湖泊科学), $2006, \mathbf{1 8}(3): 231-237$

http:// www. jlakes. org. E-mail: jlakes@ niglas. ac.cn

(c) 2006 by Journal of Lake Sciences

\title{
太湖水体中胶体磷含量初探”
}

孙小静 $^{1,3}$, 张战平 ${ }^{2}$, 朱广伟 ${ }^{1}$, 秦伯强 ${ }^{1 * *}$

(1: 中国科学院南京地理与湖泊研究所, 南京 210008)

(2: 浙江大学环境与资源学院, 杭州 310028)

(3: 中国科学院研究生院, 北京 100039)

摘 要: 为了探明太湖水体中胶体磷含量, 更深人认识营养盐在大型浅水湖泊中的循环过程和机理, 分别从太湖的梅梁 湾和贡湖湾两个不同类型的湖区采集水样, 利用切向流超滤方法分离出胶体物质, 对太湖水体中胶体磷含量进行了初步 研究. 结果表明: 太湖水体春季胶体磷含量在 $0.017-0.029 \mathrm{mg} / \mathrm{L}$ 间, 其中梅梁湾藻型湖区胶体磷含量范围是 $0.023-$ $0.029 \mathrm{mg} / \mathrm{L}$, 贡湖湾草型湖区胶体磷含量在 $0.017-0.022 \mathrm{mg} / \mathrm{L}$ 间. 梅梁湾水体胶体磷占总磷比例平均为 $28.6 \%$; 而贡 湖湾胶体磷占总磷比例平均达到 $39.3 \%$. 梅梁湾水体真溶解态磷含量显著高于贡湖湾水体, 达贡湖湾的 4 倍. 与澳大利 亚的 17 个湖泊相比, 太湖属胶体磷含量偏低的湖泊, 但相对于海洋的研究结果, 太湖胶体磷含量明显偏高.

关键词: 胶体; 磷; 切向流超滤; 太湖; 浅水湖泊

\section{Content of colloidal phosphorus in water of Lake Taihu, China}

SUN Xiaojing ${ }^{1,2}$,ZHANG Zhanping ${ }^{3}$,ZHU Guangwei ${ }^{1}$ \& QIN Boqiang ${ }^{1 * *}$

(1: Nanjing Institute of Geography and Limnology, Chinese Academy of Sciences, Nanjing 210008, P. R. China)

(2: College of Environmental and Resource Sciences, Zhejiang University, Hangzhou 310028, P. R. China)

(3: Graduate School of Chinese Academy of Sciences, Beijing 100039, P. R. China)

Abstract: Concentration of colloidal phosphorus in Lake Taihu, China, was determined in this paper. Lake water was sampled in April, 2005. Cross-flow filtration technique was applied to extract colloid from the water. The result shows that colloidal phosphorus concentration in Lake Taihu varied from 0.017 to $0.029 \mathrm{mg} / \mathrm{L}$. In Meiliang Bay, an algae type zone, it is $0.023-0.029 \mathrm{mg} / \mathrm{L}$; while in Gonghu Bay, a grass type zone, it is $0.017-0.022$ $\mathrm{mg} / \mathrm{L}$. The colloidal phosphorus account for $28.6 \%$ of total phosphorus ( TP) in Meiliang Bay, and account for $39.3 \%$ of total phosphorus (TP) in Gonghu Bay, respectively. The true-dissolved phosphorus in Meiliang Bay is four times of that in Gonghu Bay.

Keywords: Colloid; phosphorus; cross-flow ultrafiltration; Lake Taihu; shallow lake

胶体 (colloid) 的概念是胶体化学的创始人、英国科学家 Thomas Graham 于 1861 年首先提出的 ${ }^{[1]}$. 胶体 是物质的一种特殊分散状态, 是一种或几种物质以一定分散度分散于另一种物质中构成的多相体系 ${ }^{[2]}$. 在天然水体胶体的研究中, 通常用过滤截留的方法来获取胶体粒子. 因此使用的滤膜不同, 胶体的粒径范 围也不同. 例如, 有人把能透过 $0.45 \mu \mathrm{m}$ 滤膜且被 $1 \mathrm{KDa}$ 超滤膜截留的微粒视为胶体 ${ }^{[3]}$, 有人认为胶体颗 粒的大小为 $1-100 \mathrm{~nm}$ (按胶体颗粒的直径计), 也有人主张胶体颗粒的范围为 $1-1000 \mathrm{~nm}^{[1]} .20$ 世纪 90 年代以来, 新的胶体分离技术一一切向流超滤技术 (Cross-flow ultra-filtration, CFF) 迅速发展并成熟, 成为 分离水中胶体物质的有效方法, 在天然水胶体研究中得到广泛应用.

* 国家自然科学基金项目 (40203007)、中国科学院知识创新工程重大项目 (KZCX1 - SW - 12)、国家“十五”重大科 技专项 (863 计划) (2002AA601011) 共同资助. 2005-06-30 收稿;2005-09-14 收修改稿. 孙小静, 女, 1977 年 生, 在读博士. E-mail: xjsun@ niglas. ac. cn.

** 通讯作者, E-mail: qinbq@ niglas. ac.cn. 
国内外天然水胶体的研究中, 有关海洋胶体的研究较多, 通常是利用切向流超滤技术并结合其它方 法(高速离心, 现场微滤等), 对胶体进行分级, 研究不同粒级胶体有机碳、金属元素以及氮、磷等营养元素 的含量分布特征以及胶体在元素循环中的作用 ${ }^{[4-7]}$. 对海水调查分析发现, 海洋中很多金属元素赋存于胶 体粒子中, 海洋胶体对痕量金属的生物地球化学循环具有重要作用 ${ }^{[8-14]}$. 也有一些研究涉及胶体对元素 生物利用和形态转化过程的影响, 发现海洋胶体中存在微藻生长所必需的 N、P、Fe 等营养元素, 对微藻生 长有显著的刺激作用. 在适宜光合细菌生长的条件下, 海洋胶体对光合细菌生长也有显著的促进 作用 ${ }^{[15-17]}$.

与海洋相比, 湖泊水体较浅, 单位体积水体拥有水土界面面积大, 风浪对湖泊沉积物扰动作用强烈, 水体生态系统复杂; 营养盐在生态系统中的循环机制复杂, 循环速率也较快, 因而胶体的来源及其对于元 素循环的作用也更为复杂. 根据海洋胶体的研究结果 ${ }^{[15-17]}$, 湖泊胶体对湖泊生态系统中的微藻和细菌等 生物可能也存在着重要作用, 一些湖泊藻类水华的暴发很可能与水体胶体营养盐含量较高, 利于藻类短时 间内迅速生长有关, 但是国际上对湖泊胶体的研究很少, 只有少量处于起步阶段的研究, 例如, Shankar Chellam 等人在 Houston 湖研究了水体离子浓度对水中胶体颗粒稳定性的影响 ${ }^{[18]}$. Aidong Zhang 等(2001) 研究了澳大利亚西南部的 Swan 滨海平原上 17 个浅水湖沼的胶体磷含量 ${ }^{[19]}$.

大型浅水湖泊中频繁的底泥再悬浮和蓝藻水华暴发, 以及复杂的外源和多样的生态系统结构, 使得 浅水湖泊中胶体的来源、物质组成、环境行为等更加复杂, 也说明浅水湖泊胶体可能会对营养盐生物地球 化学循环过程产生重要影响. 然而目前国际上对大型浅水湖泊水体中胶体的研究仍属空白, 因此本文以 我国第三大淡水湖一太太湖为研究对象, 在两个不同的湖区定点采集水样, 对太湖中胶体磷含量进行了 初步研究, 以期为进一步探究营养盐在大型浅水湖泊中的循环过程和机理提供新的思路.

\section{1 实验材料与方法}

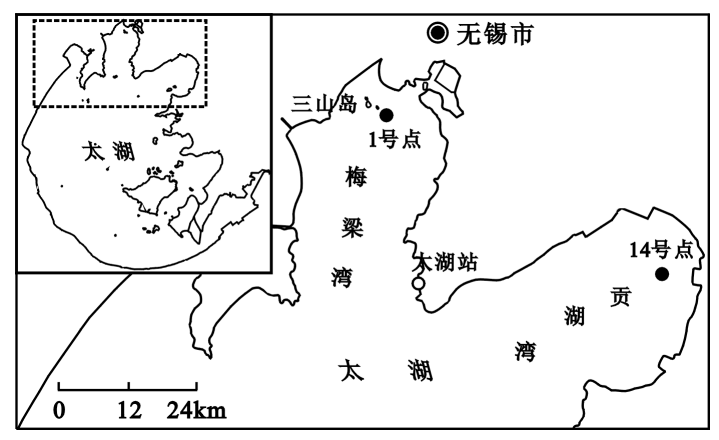

图 1 采样点位置示意图

Fig. 1 Location of the sampling sites

水样采集点选在太湖站常规采样点的 1 号点 (31. $51317^{\circ} \mathrm{N}, 120.19067^{\circ} \mathrm{E}$ ) 和 14 号点 $\left(31.43511^{\circ} \mathrm{N}, 120.37691^{\circ} \mathrm{E}\right.$ ) (图 1), 其中 1 号点位 于梅梁湾的三山岛附近, 14 号点位于贡湖湾内. 根 据中国科学院太湖湖泊生态系统研究站对两点多 年的生态监测 (1), 两点 2001-2003 年的水体叶绿素 $\mathrm{a}(\mathrm{Chl}-\mathrm{a})$ 、总磷 (TP) 月含量变化如图 2, 由图 2 可 知, 梅梁湾的 Chl-a 和 TP 含量均显著高于贡湖湾水 体同期的含量. 且有监测表明梅梁湾无水草生长, 近年来该区水华暴发严重, 属典型藻型湖区; 贡湖 湾水草生长密集, 属典型草型湖区.

于 2005 年 4 月 $15-17$ 日连续采样, 采样深度 为水表面以下 $20 \mathrm{~cm}$. 用小型直流㫤抽取 $20 \mathrm{~L}$ 湖水到氟化聚乙烯瓶中, 瓶口蒙 $64 \mu \mathrm{m}$ 笁网以过滤悬浮杂质 和大型藻类. 采样和测试用到的水样容器均经过 $10 \%$ 盐酸溶液浸泡和去离子水清洗. 水样采回后立即分 出 $5 \mathrm{~L}$ 用于测悬浮固体含量 $(\mathrm{SS}) 、 \mathrm{Chl}-\mathrm{a}$ 含量和收集悬浮物, 剩下的 $15 \mathrm{~L}$ 水样依次通过装 $25 \mu \mathrm{m}$ 滤芯 (型号 CR2571006, PP 聚丙烯材料) 和 $1 \mu \mathrm{m}$ 滤芯 (型号 CR0171006, 聚丙烯材料) 的预过滤系统 (10inch Millipore prefilter), 预滤液用于切向流超滤. 超滤系统为 Millipore Standard Pellicon System. 膜为孔径 $1 \mathrm{KDa}$ (相当于 1 $\mathrm{nm}$ ) 的 Millipore PLAC 超滤膜堆. 因此本文中的胶体是能透过 $1 \mu \mathrm{m}$ 滤膜且被 $1 \mathrm{KDa}$ 超滤膜阻止的微粒. 超 滤前, 先取 1-2L 预滤液在超滤系统中循环约 $10 \mathrm{~min}$ 后弃去, 以润洗超滤膜和管道, 其余水样进行超滤. 水样经过超滤处理后, 所得到的保留液是某种粒径胶体粒子被浓缩若干倍数的胶体浓缩液, 而透过液是 不含该粒径胶体粒子的超滤液 ${ }^{[4]}$. 水样的超滤浓缩系数为: 

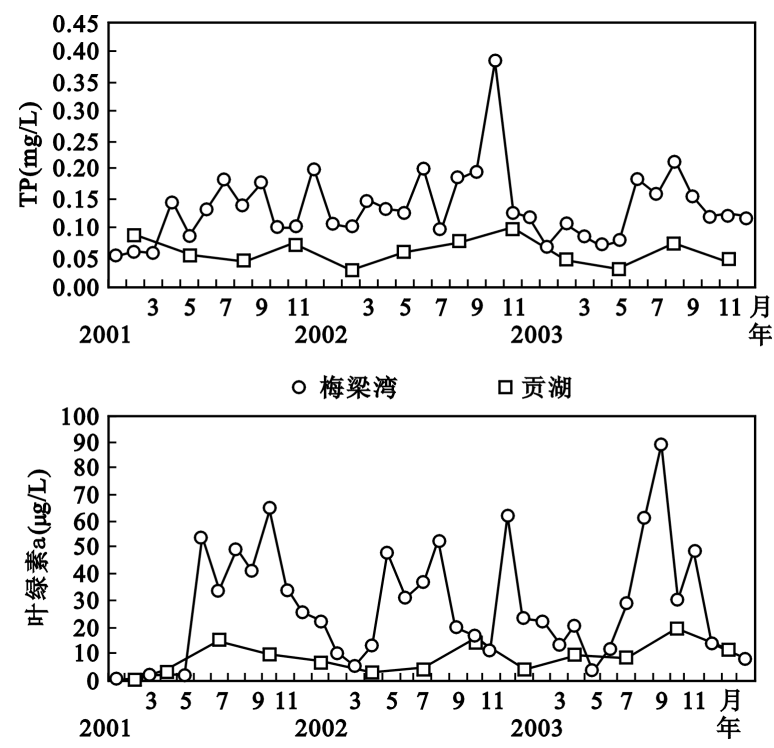

图 2 采样区 $2001-2003$ 年 TP 和 Chl-a 的逐月变化

Fig. 2 Concentrations of TP and Chl-a in water of the two sample sites from 2001 to 2003

$$
F=V_{p} / V_{r}
$$

其中, $V_{p}$ 为超滤前预滤液体积, $V_{r}$ 为超滤后保留液的体积, $F$ 为浓缩系数.

收集水样经超滤后得到的保留液和超滤液各 $100 \mathrm{ml}$, 加人数滴浓硫酸, 在 $4^{\circ} \mathrm{C}$ 下密封保存, 用于磷含 量的测定.

切向流超滤流程可参见王江涛 ${ }^{[20]}$, 每次水样超滤前后都要对超滤系统进行清洗, 清洗流程见图 3.

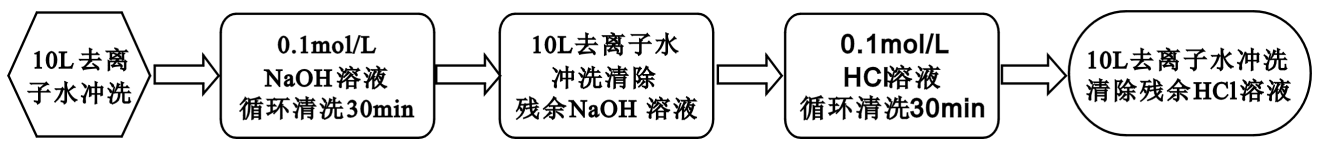

图 3 超滤系统清洗流程图

Fig. 3 Rinsing procedure of CFF system

本文中的 TP 是指湖水经 $64 \mu \mathrm{m}$ 笁网过滤后水样中总磷含量; 胶体磷是指水样中能通过 $1 \mu \mathrm{m}$ 预滤膜 且被 $1 \mathrm{KDa}$ 超滤膜截留的胶体物质中的磷含量; 真溶解态磷是指水样经 $1 \mathrm{KDa}$ 超滤膜超滤后超滤液中的磷 含量; 总溶解磷 (TDP) 则是指水样经 $1 \mu \mathrm{m}$ 滤膜过滤后透过液中的磷含量. 水样中磷含量的测定采用过硫 酸钾消解后钼锑抗比色分光光度法 ${ }^{[21]}$.

\section{2 实验结果}

\section{1 空白试验}

为保证试验结果的可靠性, 在水样超滤前, 首先用 $5 \mathrm{~L}$ 的 Milli-Q 水在切向流超滤系统中循环 $60 \mathrm{~min}$, 得到保留液 $2 \mathrm{~L}$, 在此过程中分别收集 $100 \mathrm{ml}$ 各部分水样, 用于测定磷含量. 空白样、保留液和超滤液的磷 含量分别为 $0.000 \mathrm{mg} / \mathrm{L}, 0.005 \mathrm{mg} / \mathrm{L}$ 和 $0.000 \mathrm{mg} / \mathrm{L}$, 超滤过程引人的污染在测定方法的误差允许范围内, 因此可认为对磷含量的测定结果基本没有影响. 


\section{2 超滤回收率}

样品超滤回收率 $R$ 可表示为

$$
R=\frac{C_{u} \times V_{u}+C_{r} \times V_{r}}{C_{p} \times V_{p}} \times 100 \%
$$

其中, $C_{p}$ 为预滤液中的参量浓度, $C_{u}$ 为超滤液中的参量浓度, $C_{r}$ 为保留液中的参量浓度, $V_{p}$ 为预滤液体 积, $V_{u}$ 为超滤液体积, $V_{r}$ 为保留液体积.

本研究中 6 个测定水样的磷回收率见表 1 . 由表 1 可见, 样品的磷回收率在 $81.3 \%-121.8 \%$ 之间, 这 一结果基本在文献报道的范围内 ${ }^{[22,23]}$, 表明在超滤过程中的污染或损失不明显, 磷可以通过切向流超滤 试验定量地回收.

表 1 梅梁湾和贡湖湾水样的超滤浓缩倍数和回收率

Tab. 1 Recovery of lake water samples in ultra-filtration experiments

\begin{tabular}{|c|c|c|c|c|c|c|}
\hline & \multicolumn{3}{|c|}{ 梅梁湾水样 } & \multicolumn{3}{|c|}{ 贡湖湾水样 } \\
\hline & 4 月 15 日 & 4 月 16 日 & 4 月 17 日 & 4 月 15 日 & 4 月 16 日 & 4 月 17 日 \\
\hline 预滤液体积( L) & 10 & 10 & 9 & 9 & 9 & 9 \\
\hline 保留液体积( L) & 0.58 & 0.69 & 0.53 & 0.57 & 0.59 & 0.58 \\
\hline 超滤液体积( L) & 9.42 & 9.31 & 8.47 & 8.43 & 8.41 & 8.42 \\
\hline 浓缩倍数 & 17.2 & 17.0 & 15.3 & 14.5 & 15.8 & 15.5 \\
\hline 磷的超滤回收率 & $85.1 \%$ & $108.7 \%$ & $87.7 \%$ & $92.0 \%$ & $81.3 \%$ & $121.8 \%$ \\
\hline
\end{tabular}

\section{3 不同形态磷的含量及其在 TP 中的分配}

表 2 列出了本次试验得到的两湖区不同形态磷含量的测定结果. 由表 2 可知, 梅梁湾水体胶体磷含量 范围为 $0.023-0.029 \mathrm{mg} / \mathrm{L}$, 贡湖湾水体胶体磷含量为 $0.017-0.022 \mathrm{mg} / \mathrm{L}$. 梅梁湾水体胶体磷占 TP 的比 例为 $20.8 \%-33.1 \%$, 平均为 $28.6 \%$; 而贡湖湾胶体磷占 TP 的比例为 $33.6 \%-44.8 \%$, 平均为 $39.3 \%$. 将两湖区的结果比较后可发现, 虽然梅梁湾水体的胶体磷含量略高于贡湖湾水体, 但是胶体磷占 TP 的比 例却是贡湖湾显著高于梅梁湾.

表 2 春季两湖区的不同形态磷含量

Tab. 2 Phosphorus concentration in different fractions in Meiliang Bay and Gonghu Bay

\begin{tabular}{|c|c|c|c|c|c|c|}
\hline \multirow{2}{*}{ 磷含量 $(\mathrm{mg} / \mathrm{L})$} & \multicolumn{3}{|c|}{ 梅梁湾 } & \multicolumn{3}{|c|}{ 贡湖湾 } \\
\hline & 4 月 15 日 & 4 月 16 日 & 4 月 17 日 & 4 月 15 日 & 4 月 16 日 & 4 月 17 日 \\
\hline 胶体态磷 $(1 \mathrm{KDa}-1 \mu \mathrm{m})$ & 0.023 & 0.029 & 0.027 & 0.019 & 0.022 & 0.017 \\
\hline 真溶解态磷 ( $\leqslant 1$ KDa) & 0.032 & 0.020 & 0.024 & 0.001 & 0.009 & 0.009 \\
\hline $\mathrm{TP}$ & 0.109 & 0.087 & 0.083 & 0.043 & 0.055 & 0.051 \\
\hline 胶体磷/TP & $20.8 \%$ & $33.1 \%$ & $31.9 \%$ & $44.8 \%$ & $39.6 \%$ & $33.6 \%$ \\
\hline 胶体磷/TDP & $35.4 \%$ & $57.7 \%$ & $48.7 \%$ & $93.1 \%$ & $56.8 \%$ & $79.1 \%$ \\
\hline
\end{tabular}

由图 4 可知, 梅梁湾水体 $\mathrm{TP}$ 和真溶解态磷的平均含量显著高于贡湖湾, 胶体磷的平均含量也略高于 贡湖湾. 梅梁湾水体 TP 是贡湖湾的近 2 倍, 真溶解态磷含量是贡湖湾的 4 倍, 胶体磷含量则平均只有贡 湖湾水体的 1.3 倍. 磷酸根是真溶解态磷的主要成分之一, 是藻类可直接利用磷. 梅梁湾水体真溶解态磷 显著高于贡湖湾水体, 可能是该湖区藻华暴发的原因之一.

梅梁湾水体胶体磷含量之所以没有因 TP 和真溶解态磷含量较高而显著高于贡湖湾胶体磷含量, 一方 面可能与胶体的产生方式有关, 贡湖湾水草茂盛, 水草腐烂分解时产生的腐殖质较多, 易形成大量性质稳 定的胶体物质, 胶体物质中吸附结合的磷也相应较多. 另一方面, 梅梁湾接纳太湖的主要入湖河道之

梁溪河来水, 来水携带有无锡市的城市污水, 与湖中原有水体发生混合作用, 加上来水含盐量高, 易于破坏胶体稳定, 发生聚沉作用. 而与贡湖湾相通的望虞河则是太湖的两个主要出水通道之一, 主要排 泄太湖洪水, 采样期间属于一年中湖水位较低的时期, 出湖水量小, 水流缓慢, 加上贡湖湾区水草生长茂 盛, 能起到一定的减缓水动力扰动的作用, 使得水体混和作用不强, 胶体存在状态比较稳定 ${ }^{[25,26]}$. 也有可 


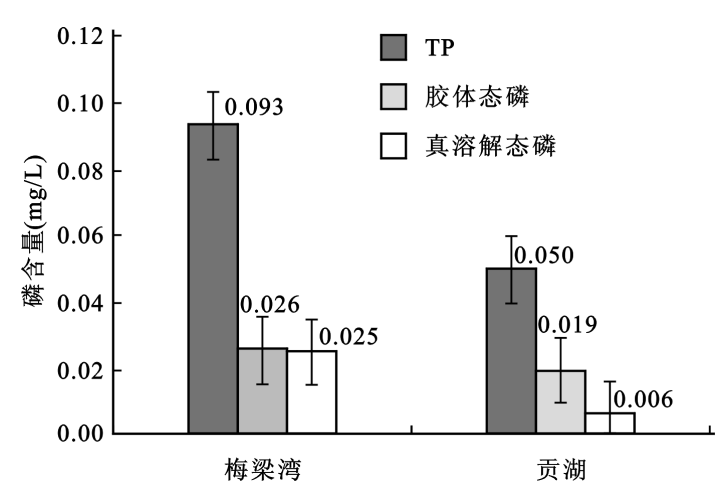

图 4 水样各形态磷含量三天测定结果的平均值

Fig. 4 Mean-value of phosphorus concentration in different fractions in three days

能是由于梅梁湾湖水的含盐量较高, 根据对中国科学院太湖湖泊生态系统研究站 $2002-2003$ 年同期水质 常规监测结果进行的统计 ${ }^{1}$, 梅梁湾同一采样点水体同期平均电导率为 $615 \mu \mathrm{S} / \mathrm{cm}$, 而贡湖湾水体同期电 导率为 $465 \mu \mathrm{S} / \mathrm{cm}$. 在含盐量较高的情况下, 胶体容易发生絮凝沉淀, 转变为颗粒态, 从而降低了胶体磷 占 TP 的百分比 ${ }^{[18,22,24]}$. 另外还有可能是由于梅梁湾水体较贡湖湾中的生物形体小且数量大, 因而营养物 质在水体生态系统中的循环速率快, 营养盐大量存在于真溶解状态和生物体中, 在作为中介的胶体颗粒 中含量较少; 而贡湖湾水体生态系统营养盐循环速率较梅梁湾慢, 很大一部分咜存于胶体颗粒中, 使得胶 体磷占 TP 的百分比较高.

\section{3 讨论}

\section{1 与国内外相关研究结果对比}

Zhang Aidong 等从澳大利亚西南部 Swan 滨海平原上的 17 个浅水永久性和季节性湖泊及沼泽取水样研 究, 发现 17 个湖泊中, 可通过 $0.50 \mu \mathrm{m}$ 滤膜的胶体磷含量在 $0.009-0.207 \mathrm{mg} / \mathrm{L}$ 间不等, 平均为 0.054 $\mathrm{mg} / \mathrm{L}$, 胶体磷占溶解性总磷 (胶体磷与真溶解态磷的总和) 的比例在 $68 \%-92 \%$ 间, 平均为 $82 \%{ }^{[19]}$. 本研 究中胶体磷含量的范围, 梅梁湾水体是 $0.023-0.029 \mathrm{mg} / \mathrm{L}$, 贡湖湾水体在 $0.017-0.022 \mathrm{mg} / \mathrm{L}$ 间, 可见与 澳大利亚的 17 个湖沼相比, 太湖属于胶体磷含量偏低的湖泊. 太湖胶体磷占可通过 $1 \mu \mathrm{m}$ 膜的溶解性总磷 含量的 35.4\%-93.1\%, 平均为 $61.8 \%$, 该比例低于文献中 $82 \%$ 的平均值, 而且分离胶体时采用的预滤 膜孔径不同, 文献中的预滤膜孔径为 $0.50 \mu \mathrm{m}$, 胶体的粒径范围是 $1 \mathrm{KDa}-0.50 \mu \mathrm{m}$, 而本研究中使用的预 滤膜孔径为 $1 \mu \mathrm{m}$, 胶体的粒径范围是 $1 \mathrm{KDa}-1 \mu \mathrm{m}$. 若本文中也使用同样 $0.50 \mu \mathrm{m}$ 孔径的滤膜分离胶体, 则胶体磷占溶解性总磷的比例会更低. 相比较而言, 太湖中胶体磷占溶解性总磷的比例也较低. 造成这一 结果的原因主要有: 文献中研究的部分湖沼本身腐殖质 (Humic substances) 含量高, 使得胶体物质总量高, 胶体磷含量相应也较高; 另外文献中提到有些湖沼属于季节性的, 雨季有水旱季干涸, 由于蒸发浓缩作 用, 采样时可能水体很浅, 营养物质含量很高, 胶体磷含量因而也较高.

赵卫红等对烟台四十里湾浅海养殖区水体胶体磷的研究发现, 该区胶体磷的浓度范围在 $0.03-0.48$ $\mu \mathrm{mol} / \mathrm{L}$ (相当于 $0.001-0.015 \mathrm{mg} / \mathrm{L}$ ) 间, 平均 $0.13 \mu \mathrm{mol} / \mathrm{L}$ (相当于 $0.004 \mathrm{mg} / \mathrm{L}$ ), 约占总溶解有机磷的 $30 \%{ }^{[23]}$. 本研究得到的太湖水体中胶体磷含量在 $0.017-0.029 \mathrm{mg} / \mathrm{L}$ 间, 高于烟台四十里湾浅海养殖区 胶体磷含量. 造成这一差别的原因可能有多个方面: 首先, 陆源物质输人对太湖水质的影响比对海洋水体 的影响大得多. 太湖的水体浅, 平均换水周期只有 $310.5 \mathrm{~d}^{[27]}$, 而海洋的换水周期可达 $2500 \mathrm{a}^{[28]}$, 大量的 陆源胶体物质 (包括腐殖质等有机胶体以及铁、锰氢氧化物等无机胶体) 和营养盐通过河道人流、水产养 殖、水土流失等输人到了太湖水体中. 这使得太湖水体的胶体物质含量显著高于海洋水体. 例如, 作为胶 
体物质主要组分之一的有机碳含量就是如此, 本次试验得出的太湖梅梁湾和贡湖湾水体胶体有机碳含量 分别为 $1.93-2.03 \mathrm{mg} / \mathrm{L}$, 而文献中浅海养殖区水体的胶体有机碳则为 $32-53 \mu \mathrm{mol} / \mathrm{L}$ (相当于 $0.38-0.64$ $\mathrm{mg} / \mathrm{L}$ ), 平均 $41.7 \mu \mathrm{mol} / \mathrm{L}$ (相当于 $0.50 \mathrm{mg} / \mathrm{L}$ ) ; 二是太湖水体的初级生产力高于浅海水体, 浮游藻类及其 它水生生物含量高, 其生命活动过程中产生的胶体物质也较多 ${ }^{[13]}$. 影响初级生产力的因素主要有水温、光 照、浮游植物生物量、营养盐浓度 ${ }^{[26]}$. 本研究的采样期间, 天气晴好, 气温为 $20-25^{\circ} \mathrm{C}$, 非常适宜浮游植 物生长, 浮游植物的生物量平均为 $49 \mathrm{mg} / \mathrm{m}^{3}$ ( 以 Chl-a 含量计), 其它营养盐如氮和碳的含量也比浅海区要 高; 三是浅海养殖区海水含盐量高于湖水, 胶体易聚沉或絮凝转变为较大的颗粒态物质, 从而使水体中胶 体物质本身的含量就小于太湖水体 ${ }^{[22,24]}$; 这些因素共同作用, 使得太湖水体的胶体磷含量较浅海区高.

\section{2 与太湖湖泊生态系统研究站 $2002-2003$ 年同期常规监测结果的比较}

根据对太湖湖泊生态系统研究站 $2002-2003$ 年水质常规监测结果进行的统计 ${ }^{1}$, 同期梅梁湾采样点 水体 $\mathrm{TP}$ 含量年平均为 $0.104 \mathrm{mg} / \mathrm{L}$, 贡湖湾采样点水体 $\mathrm{TP}$ 含量年平均为 $0.045 \mathrm{mg} / \mathrm{L}$, 本次试验得到的梅 梁湾和贡湖湾水体三天平均 TP 含量分别为 $0.093 \mathrm{mg} / \mathrm{L}$ 和 $0.050 \mathrm{mg} / \mathrm{L}$, 与常规监测结果非常接近, 从而说 明了本次采样具有很好的代表性.

表 3 本次试验和 $2002-2003$ 年常规监测的平均磷含量 $(\mathrm{mg} / \mathrm{L})$ 比较

Tab. 3 Comparison of the average phosphorus concentration between the investigation and routine survey

\begin{tabular}{ccccccccc}
\hline & \multicolumn{3}{c}{$2002-2003$ 两年平均 } & & \multicolumn{3}{c}{ 本次试验 } \\
\cline { 2 - 3 } \cline { 7 - 8 } & $\mathrm{TP}$ & $\begin{array}{c}\mathrm{TDP} \\
(\leqslant 0.45 \mu \mathrm{m})\end{array}$ & 磷酸根 & & $\mathrm{TP}$ & & $\begin{array}{c}\text { 真溶解态磷 } \\
(\leqslant 1 \mathrm{KDa})\end{array}$ & $\begin{array}{c}\text { 胶体磷 } \\
(1 \mathrm{KDa}-1 \mu \mathrm{m})\end{array}$ \\
\hline 梅梁湾 & 0.104 & 0.030 & 0.004 & & 0.093 & 0.025 & 0.026 \\
贡湖湾 & 0.045 & 0.016 & 0.005 & & 0.050 & 0.006 & 0.0194 \\
\hline
\end{tabular}

\section{4 结论}

本文首次报道了太湖水体中胶体磷含量的研究结果, 并得出以下结论:

1) 太湖水体春季胶体磷含量在 $0.017-0.029 \mathrm{mg} / \mathrm{L}$ 间, 其中梅梁湾藻型湖区胶体磷含量范围为 0.023 $-0.029 \mathrm{mg} / \mathrm{L}$, 贡湖湾草型湖区胶体磷含量在 $0.017-0.022 \mathrm{mg} / \mathrm{L}$ 间. 梅梁湾水体胶体磷占 $\mathrm{TP}$ 的比例平 均为 $28.6 \%$; 而贡湖湾胶体磷占 TP 的比例平均达 $39.3 \%$.

2) 梅梁湾水体 TP 含量是贡湖湾的近 2 倍, 真溶解态磷含量是贡湖湾的 4 倍, 胶体磷含量则平均只有 贡湖湾水体的 1.3 倍.

3) 与澳大利亚的 17 个湖泊相比, 太湖属于胶体磷含量偏低的湖泊, 但相对于海洋研究结果, 太湖的 胶体磷含量又较高.

本文得出的太湖水体胶体磷含量相当可观, 说明胶体在湖泊营养盐循环中可能起着重要作用, 关于 太湖中胶体和胶体态营养盐的特征和其对生态系统的作用还需要继续开展研究, 以期更深人认识营养盐 的生物地球化学循环规律, 为控制污染、消除水华带来新的思路和方法.

致谢: 感谢太湖湖泊生态系统研究站提供了太湖常规生态监测数据. 王军博士在文章撰写过程中给予了宝 贵修改意见, 季江、钱荣树、罗潋葱、张运林、赵巧华、冯胜、陈永根和王艳飞同学在采样及测试过程中给予 了热情的帮助, 在此一并表示感谢.

\section{5 参考文献}

[1] 沈 钟, 王果庭编著. 胶体与表面化学(第二版). 北京: 化学工业出版社, 1997: 1-104.

[2] 董元彦, 李宝华, 路福绥主编. 物理化学. 北京:科学出版社, 2001: 229-230.

[3] Orjan G, Philip M G. Aquatic colloids: Concepts, definitions, and current challenges. Limnol \& Oceanogr $1997, \mathbf{4 2}(3): 519-528$.

[4] 赵新淮, 张正斌, 刘莲生. 天然水体中的胶体粒子. 黄渤海海洋, 2001, 19 (2): 107 - 114. 
５］ 赵卫红. 海洋中胶体研究的新进展. 海洋与湖沼, 2000, 31(2) : $221-229$.

[6] 李 丽, 张正斌, 刘莲生等. 南黄海胶体有机碳和溶解有机碳的分布. 青岛海洋大学学报, 1999, 29 (2) : $321-324$.

[7] 赵卫红, 崔 金金, 王江涛. 切向超滤技术分离海洋胶体有机碳性能的研究. 自然科学进展, 2004, 14 (12) : $1447-1451$.

[8] 魏俊峰, 戴民汉, 洪华生等. 海洋胶体与痕量金属的相互作用. 地球科学进展, 2004, 19(1): 26-31.

[9] 陈 敏, 黄奕普, 丘雨生. 从 ${ }^{234} \mathrm{Th}$ 的固/液分配看海洋胶体的作用. 海洋与湖沼, 1999, 30 (6): 726 -730 .

[10] Honeyman J T, Santschi P H. A “Brownian-pumping” model for oceanic trace metal scavenging: evidence from Th isotopes. Mar Res, 1989, (47): $951-992$.

[11] Dai M H, Martin J M, Cauwet G. The significant role of colloids in the transport and transformation of organic carbon and associated trace metals $(\mathrm{Cd}, \mathrm{Cu}$ and $\mathrm{Ni}$ ) in the Rhi ne delta (France). Marine Chemistry, $1995,(51): 159-175$.

[12] Dai M H, Buesseler K O, Kelley J M. Size-fractionated plutonium isotopes in a coastal environment. Journal of Environmental Radioactivity, 2001, (53): 9 - 25.

[13] Ingri J, Nordling S, Larsson J, et al. Size distribution of colloidal trace metals and organic carbon during a coastal bloom in the Baltic Sea. Marine Chemistry, 2004, (91): 117 - 130.

[14] Wells M L, Smith G J, Bruland K W. The distribution of colloidal and particulate bioactive metals in Narragansett Bay, RI. Marine Chemistry, 2000, (71) : $143-163$.

[15] 赵新淮, 张正斌, 韩喜江. 海水胶体与 $\mathrm{PO}_{4}^{3-} 、 \mathrm{Cu}^{2+}$ 的作用及对微藻生长的影响. 哈尔滨工业大学学 报, 2002, 34(6): $776-779$.

[16] 郑爱蓉, 陈 敏, 吕 娥等. 海洋胶体中的氮、磷和铁对微藻生长的效应. 自然科学进展, 2004, 14 (3) : $339-343$.

[17] 郑爱蓉, 陈 敏, 郑雪红等. 海洋胶体对光合细菌生长的效应. 自然科学进展, 2002, 12(7):759 -762 .

[18] Shankar C, Jiang T, Ramesh S. Characterization of natural colloidal and organic materials in Lake Houston. Annual Report of Environmental Institute of Houston, 2000: 19 - 21.

[19] Zhang A D, Oldham C. The use of an ultrafiltration technique for measurement of orthophosphate in shallow wetlands. The Science of the Total Environment, 2001, (266) : $159-167$.

[20] 王江涛. 黄河、长江和钱塘江水体中的胶体有机碳. 科学通报, 1998, 43(8): $840-843$.

[21] 国家环境保护总局, 水和废水监测分析方法编委会编著. 水和废水监测分析方法. 北京: 中国环境科 学出版社, 2002: $243-248$.

[22] Dai M H, Martin J M, Hong H S, et al. Preliminary study on the dissolved and colloidal organic carbon in the Zhujiang River estuary. Chinese Journal of Oceanology and Limnology, 2000, 18(3) : 265 - 273.

[23] 赵卫红, 王江涛. 烟台四十里湾养殖区的胶体有机碳、氮和磷. 济南大学学报 (自然科学版), 2004, 18(2) : $132-135$.

[24] Gunnars A, Blomqvist S, Johansson P, et al. Formation of Fe( III) oxyhydroxide colloids in freshwater and brackish seawater, with incorporation of phosphate and calcium. Geochimica et Cosmochimica Acta, 2002 , 66(5): $745-758$.

[25] 孙顺才, 黄渏平主编. 太湖. 北京: 海洋出版社,1993: 19-20.

[26] 秦伯强, 胡维平, 陈伟民等编著. 太湖水环境演化过程与机理. 北京:科学出版社, 2004: 9-33, 248 -253 .

[27] 王苏民, 窦鸿身主编. 中国湖泊志. 北京:科学出版社, 1998: 60 .

[28] 朱颜明, 何 岩等编著. 环境地理学导论. 北京: 科学出版社, 2002: 56-59. 